\title{
Wideband signal detection for cognitive radio applications with limited resources

\author{
Shaoyang Men ${ }^{1}$, Pascal Chargé2 ${ }^{2}$ Yide Wang ${ }^{2,3}$ and Jianzhong Lii*
}

\begin{abstract}
Wideband signals are expected to be used to achieve the required quality of service (QoS) in the next generation of wireless communications, civil and military radar, and many wireless sensor network (WSN) scenarios. Wideband signal detection has been identified as one of the most challenging problems in the proliferation of the cognitive radio technology. Moreover in many applications, spectrum sensing in cognitive radio (CR) is expected to be performed with limited resources in terms of time, computation, and complexity. This paper is dedicated to the detection of a wideband signal with small sample size. Aiming at using small sample size, a statistical model of samples is given based on Student's $t$ distribution. However, the limited number of channel observations brings a reduction of confidence in the decision. A set of new basic probability assignments associated with the hypothesis of the occupied or vacant channel are then proposed to perform the Dempster-Shafer (D-S) decision process. Simulation results show that the proposed method has much higher sensitivity to sense an occupied channel than the traditional energy detection method (ED) and the decision fusion method when small sample size is used.
\end{abstract}

Keywords: Wideband signal detection, Cognitive radio, Small sample size, Dempster-Shafer theory of evidence

\section{Introduction}

With the evolution and development of various wireless technologies, spectrum resources are becoming scarce due to the increasing need for spectral bandwidth and number of users. Cognitive radio (CR) technology has attracted a lot of interest, especially for the next generation of wireless communications, many types of radar systems and wireless sensor network (WSN) [1-4]. In all those systems, wideband signals are expected to be used to achieve the required quality of service (QoS). Therefore, wideband signal detection plays an important role in a wide range of wireless communication systems and has been identified as one of the most challenging problems in the CR technology applications [5-7].

Although, there are numerous current research works focusing on wideband signal detection, many severe challenges still exist $[8,9]$. First of all, in realistic scenarios, it is very difficult to know the number of antennas, the coding scheme, and the structure of the detected signal. Therefore, an accurate blind spectrum sensing method

\footnotetext{
*Correspondence: jianzhong.li@gdut.edu.cn

${ }^{4}$ School of Automation, Guangdong University of Technology, No. 100,

Waihuan West Road, Panyu District, 510006 Guangzhou, China
}

Full list of author information is available at the end of the article without any prior information is of great interest. Moreover, in order to avoid unexpected harmful interference, CR user must be able to quickly vacate the frequency band when the licensed user starts transmitting. Thus, the sensing time must be limited to an acceptable level, while still guarantee a sufficiently low detection error probability.

For this purpose, a number of spectrum sensing methods have been proposed and investigated in [10-17]. Under no prior knowledge about the wideband signal, energy detection (ED) has been shown to be the most popular technique in cooperative sensing thanks to its low computational power requirements on wireless devices $[3,13]$. However, energy detection is limited by the signalto-noise ratio (SNR) wall and has high probability of false alarm [14]. In order to overcome these shortcomings, eigenvalue-based spectrum sensing methods have been proposed $[14,15]$, which are mainly based on the asymptotic or limiting distribution of extreme eigenvalues in order to overcome the noise uncertainty problem. Unfortunately, they cannot be extended to a more general dimensional setting due to their daunting computational cost. Moreover, these techniques require large number of samples, which is often not suitable for real application scenarios [16]. Thus [18-20], study the spectrum sensing 
method using goodness-of-fit (GoF) test for small sample size, relying on the Anderson-Darling (AD) statistic. In that case, the GoF test is only performed to assess the rejection (or not) of the null hypothesis (i.e., the absence of PU signal). Different from the GoF test mentioned above, both hypotheses of the presence and absence of the wideband signal would be considered in the proposed method in order to make full use of the statical information of the binary hypotheses and improve the detection performance.

Considering the challenges mentioned above, a robust spectrum sensing method with small sample size is proposed in this work. On the one hand, this means short time in real-time data processing. Especially when the detecting devices have only a single-radio architecture, the time of sampling and observing the channel is expected to be as short as possible. On the other hand, we consider that only less steady state reception can be obtained in some complex information environment. Firstly, considering that the Gaussian approximation in ED is good only when the sample size is sufficiently large [21], we reformulate the spectrum sensing into Student's $t$ distribution test problem as in [19], which is popular in situations where the sample size is small. Besides, based on the characteristics of Student's $t$-distribution, new basic probability assignment (BPA) functions are proposed for estimating the presence or not of a wideband signal emitter. However, due to the small number of samples, the estimation performance inevitably suffers from lack of reliability. In order to improve the reliability, Dempster-Shafer (D-S) theory of evidence [22-30] is used to make a final decision. As in [31], in the proposed method, Student's $t$ distribution of a reduced number of samples is used. The main contribution stands in the proposition of two new BPA functions to evaluate the credibility of the collected small samples from a wideband signal and the combination of BPA functions in order to make a more reliable decision. Specifically, the novelty is that the cumulative distribution function (CDF) of Student's $t$ distribution is utilized to define the BPA functions, which is different from the BPA functions based on the CDF of normal distribution and ED in [23-27]. The new BPAs based on Student's $t$ distribution are more appropriate for dealing with the situation of small samples than the BPAs based on normal distribution. Moreover, in the proposed scheme, in order to fully exploit the collected samples, both hypotheses of presence or absence of wideband signal are used. Simulations show that the proposed method has much higher sensitivity to detect the presence of a signal than ED- and GoF-based methods.

The rest of the paper is organized as follows. In Section 2, some spectrum sensing preliminaries are presented. The proposed spectrum sensing scheme including the statistical model of the received small samples, basic probability assignment functions and D-S fusion, is described in Section 3. Simulation results and conclusions are given in Sections 4 and 5, respectively.

\section{Spectrum sensing preliminaries}

In this paper, we assume that a wideband signal needs to be detected. According to [32], a signal having a fractional bandwidth greater than 0.01 and smaller than 0.25 is categorized as wideband. Consider that the observed bandwidth is subdivided into $K$ subbands with equal

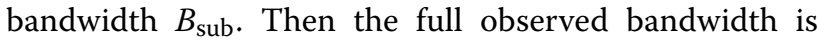
$B_{\text {tot }}=K B_{\text {sub. In each subband, signals are band-passed }}$ and downconverted to the baseband. In order to provide a detection in a very short time, a limited number of real-valued samples $Q$ are collected in each subband. The Nyquist rate in each subband is $2 B_{\text {sub }}$, and by setting the oversampling factor to $N$, the sampling period is $T_{s}=\frac{1}{2 B_{\mathrm{sub} N}}$. In each subband, the signal is oversampled with a factor $N$, which means that the sampling frequency is very much larger than the subband width. Actually, when $N$ is large enough while maintaining a small $Q$ (number of samples), the scheme in each subband can be seen as a narrowband signal sampling process. As the over sampling factor $N$ is increased, the observation duration $Q T_{s}$ is reduced. Let $x_{q}^{(k)}$ be the $q$ th sample in the $k$ th subband. Wideband signal detection can be formulated as a binary hypothesis problem as follows

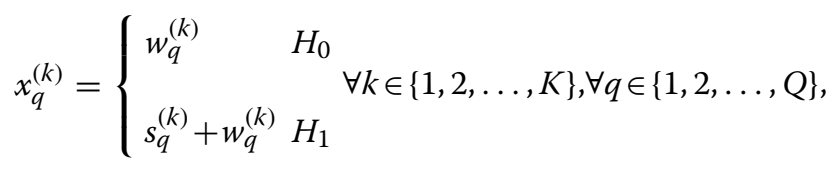

where $H_{0}$ and $H_{1}$ respectively represent the hypotheses of absence and presence of a signal in the $k$ th subband. $w_{q}^{(k)}$ is the noise contribution in the sample. Without loss of generality, we assume that the noise is an additive white Gaussian noise (AWGN) with zero mean and variance $\sigma^{2}$. $s_{q}^{(k)}$ is the signal component in the $k$ th subband when it is present. According to the oversampling assumption and small number of samples (short observation), it can be assumed that the signal is constant during its observation so that $s_{q}^{(k)}=s^{(k)}$. In each of the $K$ subbands, $Q$ samples are collected with oversampling factor $N$. The more $K$ is large, the more the narrowband signal hypothesis in each subband is true. The more $K$ and $N$ are large, the more the constant signal assumption over $Q$ samples tends to be true. Since the number of samples $Q$ is small, the observation duration is very short and during this short period, the narrowband signal can be approximated as constant. Moreover, in practice, the distribution of the power spectral density of the signal is unknown, we assume that the signal uniformly occupies the full bandwidth which is the most reasonable, fair, and neutral assumption. As an 
example, this assumption holds in many multicarrier signals schemes. It allows to model the signal as a constant in both time and frequency domains. Let us consider in this study that $s^{(k)}=1$, without loss of generality. In the simulations section below, the values of $K, N$, and $Q$ have been selected arbitrarily as a matter of example, and some simulations are provided in Subsection 4.4 to support the signal assumptions.

In this case, the spectrum sensing problem is equivalent to a standard scenario with Gaussian distributions having equal variance and different means under each hypothesis.

\section{Methods}

The proposed spectrum sensing method relies on a fusion processing using D-S theory and a new set of BPA functions. BPA definition and evaluation are the key points of the D-S fusion. In most applications, it is generally assumed that the number of available samples is sufficiently large in order to correctly estimate the BPAs and perform a reliable fusion. But in this work, we consider that the CR device is very limited in terms of sample size. Hence, we propose to define some new BPAs relying on Student's $t$ distribution.

\subsection{Statistical model of the received small samples}

Considering the small number of samples and the special sensing scenario about detecting a wideband signal in a zero mean Gaussian noise, it is shown that the optimal test in signal detection is Student's $t$ test [31]. In order to construct the test statistic in accordance with Student's $t$ distribution, we denote, respectively, $X_{k}$ and $S_{k}^{2}$ as the mean and variance of the samples in the $k$ th subband,

$$
X_{k} \triangleq \sum_{q=1}^{Q} \frac{x_{q}^{(k)}}{Q}
$$

and

$$
S_{k}^{2} \triangleq \sum_{q=1}^{Q} \frac{\left(x_{q}^{(k)}-X_{k}\right)^{2}}{Q-1}
$$

where $k=1,2, \cdots, K$. Hence, $K$ variables (one from each subband) are obtained as

$$
Y_{k} \triangleq \frac{X_{k} \sqrt{Q}}{S_{k}}, \quad k=1,2, \cdots, K
$$

Under $H_{0}$ hypothesis, there exists only noise, $x_{q}^{(k)} \sim$ $\mathcal{N}\left(0, \sigma^{2}\right)$, then $Y_{k}$ follows Student's $t$ distribution with degree $v=Q-1$ degrees of freedom. Otherwise, under $H_{1}$ hypothesis, the received signal samples include the wideband signal and noise, then $x_{q}^{(k)} \sim \mathcal{N}\left(\mu, \sigma^{2}\right)$, with $\mu=1$ in our case. It comes that $Y_{k}$ has a non-central Student's $t$ distribution with $v=Q-1$ degrees of freedom and non-centrality parameter $\delta=\sqrt{Q \mu^{2} / \sigma^{2}}$, where $\mu^{2} / \sigma^{2}$ is the SNR [19].

The probability density function (PDF) of Student's $t$ distribution has the similar bell shape of a normally distributed variable with mean 0 and variance 1 , except that it is a bit lower and wider. The larger $Q$ is, the more Student's $t$ distribution approaches the standard normal distribution [33]. Conversely, when $Q$ is small, the tails of Student's $t$ distribution are much heavier than those of the normal distribution, as shown in Fig. 1. Moreover, the PDFs of the non-central $t$ distribution with different $Q$ are also given in Fig. 1. As we can see, there is a certain overlap between the tails of the $t$ distribution and the non-central $t$ distribution with the same degrees of freedom $v=Q-1$, and the overlapping region decreases with the increase of $Q$ from Fig. 1a to d. This also validates that $Y_{k}$ in Eq. (4) for small $Q$ is prone to taking values that fall far from their statistical mean and leads to an unreliable BPA estimation. Therefore, we propose to calculate $K$ variables $Y_{k}$ and combine them by D-S theory of evidence for a reliable decision. In addition, in order to estimate the belief of the observed signal in each subband, the cumulative distribution functions (CDF) of $Y_{k}$ under $H_{0}$ and $H_{1}$ denoted by $F_{0}(y)$ and $F_{1}(y)$ are applied, which are given in [33]. For D$\mathrm{S}$ fusion, we assume that $Y_{k}$ has at least two values, that is $K \geq 2$.

Note that in this statistical model we reformulate the received samples $x_{q}^{(k)}$ into a new variable $Y_{k}$, which has Student's $t$ distribution and non-central $t$ distribution under $H_{0}$ and $H_{1}$ hypotheses, respectively. The CDF $F_{0}(y)$ of $Y_{k}$ under $H_{0}$ hypothesis only depends on the degrees of freedom $v$, while $F_{1}(y)$ is related to parameter $\delta=$ $\sqrt{Q \cdot \mathrm{SNR}}$. In this work, we assume that the noise variance $\sigma^{2}$ is known, as in ED-based methods. Moreover, for the proposed method, as explained in the next section, the wideband signal detection is done by evaluating the reliabilities of both $H_{0}$ and $H_{1}$ hypotheses, which is a beneficial feature that is not used in the conventional GoF test based methods.

\subsection{Basic probability assignment estimation}

According to the D-S theory of evidence [22], $\Omega$ denotes the universal set, and let $2^{\Omega}$ be its power set. A function $m: 2^{\Omega} \mapsto[0,1]$ named basic probability assignment (BPA) is defined to quantify the candidate proposition as follows:

$$
m(\emptyset)=0, \quad \sum_{A \subset 2^{\Omega}} m(A)=1 .
$$

where for any set $A \subset 2^{\Omega}, m(A)>0$ which provides the degree of confidence that proposition $A$ is true. Then, in our framework, $2^{\Omega}=\left\{\emptyset,\left\{H_{0}\right\},\left\{H_{1}\right\}, \Omega\right\}$. 


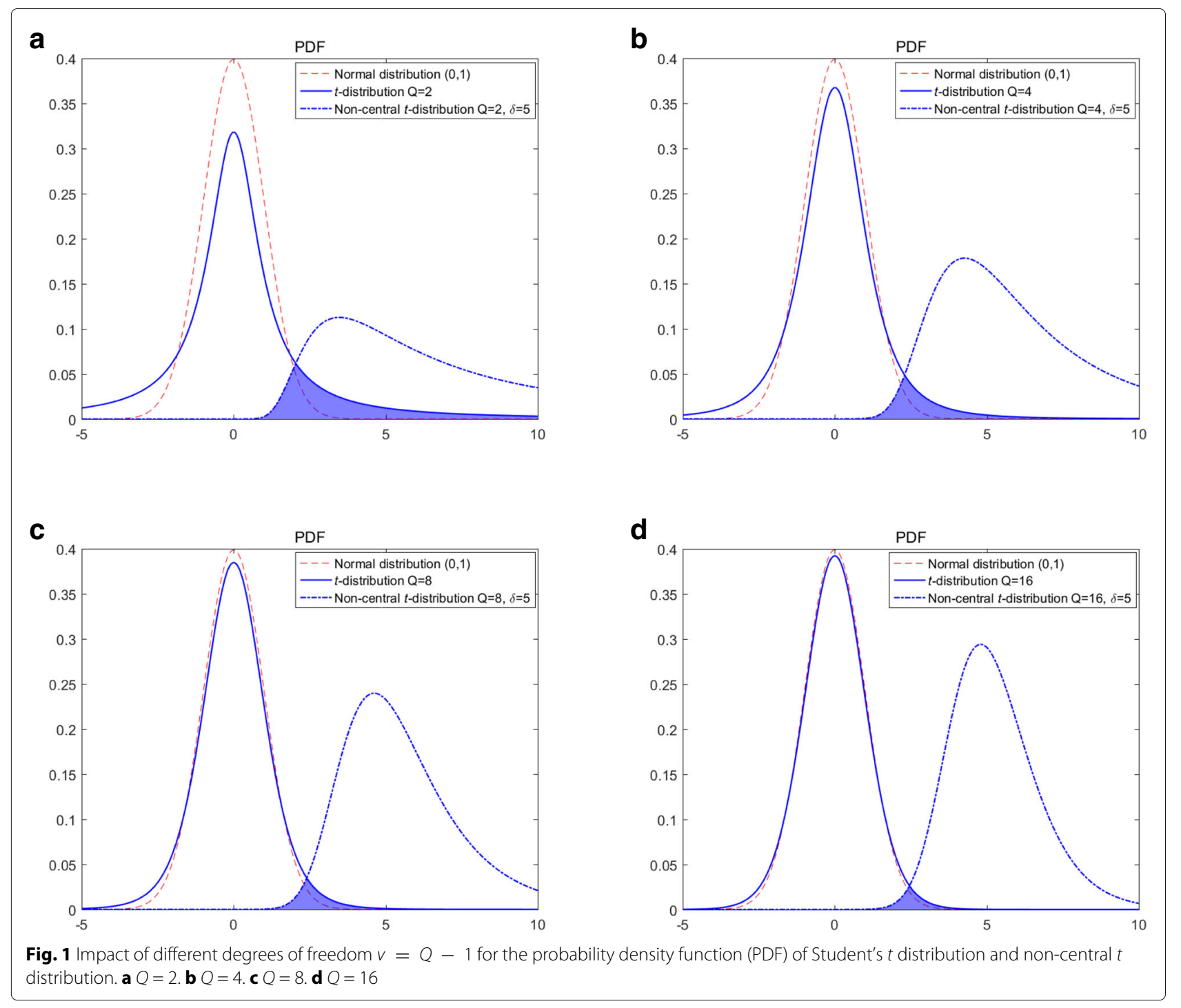

In order to evaluate the credibility of the collected samples in the $k$ th subband, we propose two new BPA functions $m_{k}\left(H_{0}\right)$ and $m_{k}\left(H_{1}\right)$ for $H_{0}$ and $H_{1}$ hypotheses in Eqs. (6) and (7), respectively

$$
\begin{aligned}
& m_{k}\left(H_{0}\right)=1-F_{0}\left(Y_{k}\right) \\
& m_{k}\left(H_{1}\right)=F_{1}\left(Y_{k}\right)
\end{aligned}
$$

where $m_{k}\left(H_{0}\right)$ and $m_{k}\left(H_{1}\right)$ are related to the CDF $F_{0}(y)$ and $F_{1}(y)$ of $Y_{k}$, respectively. Importantly, these BPA functions indicate the credibilities for hypotheses $H_{0}$ and $H_{1}$ to be true, respectively. For example, a larger value of $Y_{k}$ results in a larger $m_{k}\left(H_{1}\right)$ and a smaller $m_{k}\left(H_{0}\right)$, and vice versa, as shown in Fig. 2. Thus, we can make a decision on the presence or not of the wideband signal by comparing $m_{k}\left(H_{0}\right)$ and $m_{k}\left(H_{1}\right)$. If $m_{k}\left(H_{1}\right)>m_{k}\left(H_{0}\right)$, the signal exists; otherwise, the signal does not exist. However, since the number of samples $Q$ is small, $Y_{k}$ has been obtained

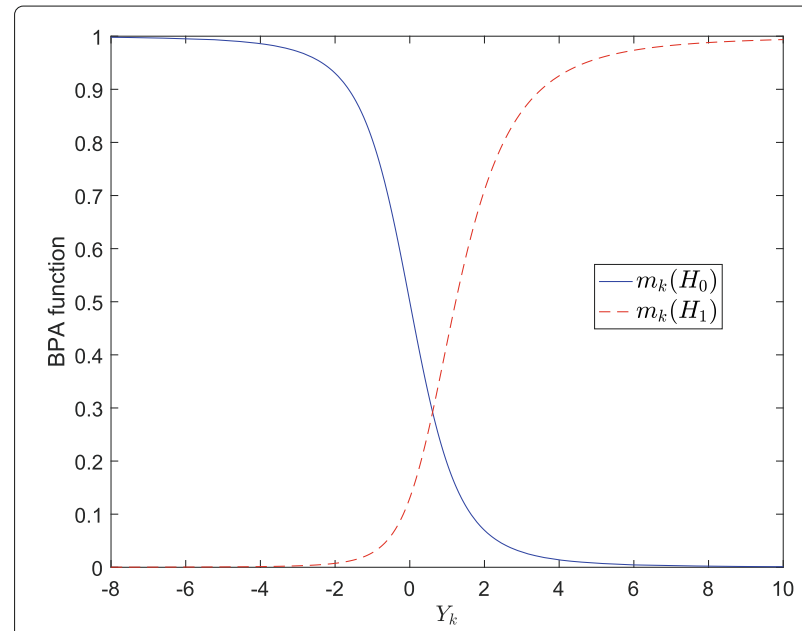

Fig. 2 Tendency of the BPA functions of $Y_{k}$ including $m_{k}\left(H_{0}\right)$ under $H_{0}$ hypothesis and $m_{k}\left(H_{1}\right)$ under $H_{1}$ hypothesis 
with a small number. This will cause a big uncertainty and increase the conflict between $m_{k}\left(H_{0}\right)$ and $m_{k}\left(H_{1}\right)$. Then a third BPA function is defined as follows:

$$
m_{k}(\Omega)=1-m_{k}\left(H_{0}\right)-m_{k}\left(H_{1}\right)
$$

where $\Omega=\left\{H_{1}, H_{0}\right\}$ denotes either hypothesis could be true and $m_{k}(\Omega)$ indicates the total uncertainty of the $k$ th subband of samples. $m_{k}(\Omega)$ is finally equal to $F_{0}-F_{1}$ by combining Eqs. (6), (7), and (8). We can show that the value of $F_{0}-F_{1}$ is non-negative with the CDFs of Student's $t$ distribution and the non-central $t$ distribution. As shown in Fig. 3, the CDF of Student's $t$ distribution $F_{0}$ (the solid line) is on the left side of the non-central $t$ distribution $F_{1}$ (the dashed line). Then, for the same value $Y_{k}, F_{0}$ must be greater than or equal to $F_{1}$. In order to improve the probability of detection and reduce the influence of the conflict evidence, we make a final reliable decision by fusing all BPA functions obtained from the $K$ groups of samples.

\subsection{D-S fusion and final decision}

In order to improve the reliability of detection, we need to combine the $K$ BPA functions and make a final decision. Then, according to the basic D-S theory of evidence and Eqs. (6), (7), and (8), two new combined BPA functions can be obtained as [23]

$$
\begin{aligned}
m\left(H_{0}\right) & =m_{1}\left(H_{0}\right) \oplus m_{2}\left(H_{0}\right) \oplus \cdots \oplus m_{K}\left(H_{0}\right) \\
& =\frac{1}{1-\kappa} \sum_{A_{1} \cap A_{2} \cap \cdots \cap A_{K}=H_{0}} \prod_{k=1}^{K} m_{k}\left(A_{k}\right) \\
m\left(H_{1}\right) & =m_{1}\left(H_{1}\right) \oplus m_{2}\left(H_{1}\right) \oplus \cdots \oplus m_{K}\left(H_{1}\right) \\
& =\frac{1}{1-\kappa} \sum_{A_{1} \cap A_{2} \cap \cdots \cap A_{K}=H_{1}} \prod_{k=1}^{K} m_{k}\left(A_{k}\right)
\end{aligned}
$$

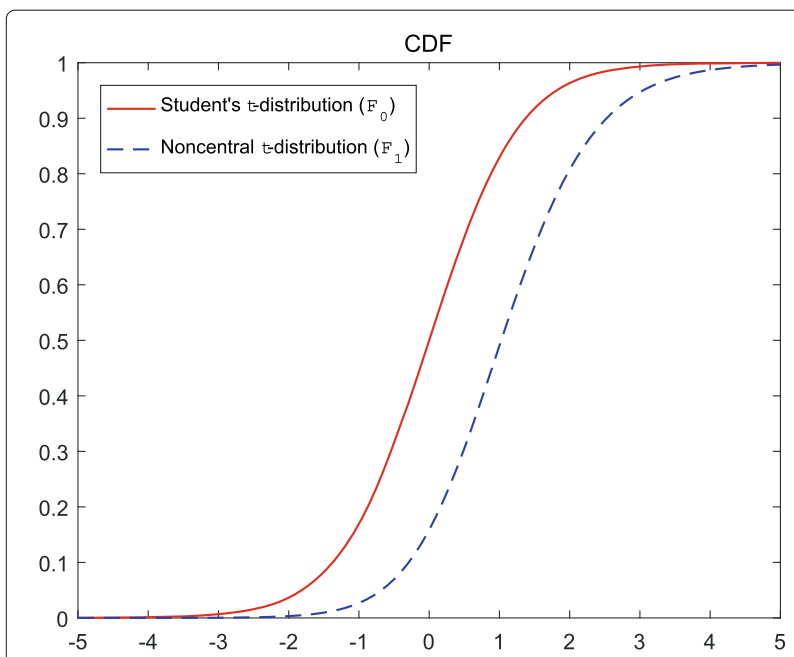

Fig. 3 Cumulative density function (CDF) of Student's $t$ distribution and the non-central $t$ distribution where $A_{k} \subset 2^{\Omega}$ for $k \in\{1, \ldots, K\}$ and $\kappa$ is a measure of the amount of conflict among the mass sets:

$$
\kappa=\sum_{A_{1} \cap A_{2} \cap \cdots \cap A_{K}=\emptyset} \prod_{k=1}^{K} m_{k}\left(A_{k}\right) .
$$

From Eqs. (9), (10), and (11), we can see that the two new BPA functions $m\left(H_{0}\right)$ and $m\left(H_{1}\right)$ are obtained by using the orthogonal sum for $K$ BPA functions, which indicate the credibilities for hypotheses $H_{0}$ and $H_{1}$ to be true, respectively. The notation $m=m_{1} \oplus m_{2}$ is called the orthogonal sum of $m_{1}$ and $m_{2}$, which is commutative and associative. If and only if there exist at least two subsets $A_{1}$ and $A_{2}$ of $2^{\Omega}$ with $A_{1} \cap A_{2} \neq \emptyset$ such that $m_{1}\left(A_{1}\right) \neq 0$ and $m_{2}\left(A_{2}\right) \neq 0$, then $m$ is calculated as shown in Eq. (12) [23].

$$
m(A)=\frac{\sum_{A_{1} \cap A_{2}=A} m_{1}\left(A_{1}\right) m_{2}\left(A_{2}\right)}{\sum_{A_{1} \cap A_{2} \neq \emptyset} m_{1}\left(A_{1}\right) m_{2}\left(A_{2}\right)} .
$$

Finally, based on all $K$ subband observations, the decision is made by comparing the ratio between $m\left(H_{1}\right)$ and $m\left(H_{0}\right)$ as follows:

$$
\frac{m\left(H_{1}\right)}{m\left(H_{0}\right)} \underset{H_{0}}{\stackrel{H_{1}}{\gtrless}} \eta
$$

where $\eta$ is the decision threshold. In fact, it is difficult to derive the closed-form expression for the threshold $\eta$ and the probability of detection for the proposed method. Therefore, we have developed a simulation model. According to the constant false alarm rate (CFAR) definition, the threshold $\eta$ corresponding to a given probability of false alarm is determined in advance by a Monte Carlo simulation with 10,000 independent runs. With the changing of $\eta$, the corresponding detection probability is determined, which is used to draw the receiver operating characteristics curves (ROC). The simulation settings and some examples are given in Section 4.

Consequently, the pseudo code of the proposed spectrum sensing method with small sample size is given in Algorithm 1. Note that the computational complexity of the proposed method mainly comes from D-S fusion (step 10 in Algorithm 1. Generally, it increases rapidly with the number of elements in the frame of discernment $(\Omega)$ and the number of the subbands $K$, as shown in Eqs. (9) and (10). However, since the frame of discernment consists of only two elements $\left\{H_{0}, H_{1}\right\}$ for the spectrum sensing, the combination of two mass functions requires the computation of $2 \times 2$ intersections [28]. Moreover, in the proposed scheme, due to the division of the observed bandwidth into $K$ subbands, a large $K$ increases the number of BPA functions then the computational complexity of the D-S fusion. 


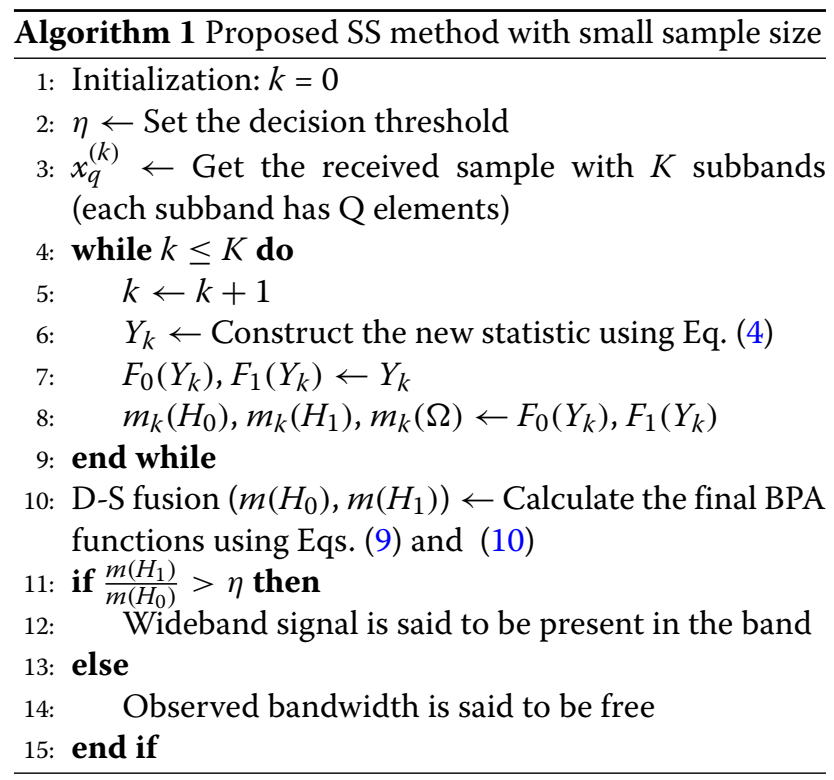

\section{Simulation results and analysis}

In this section, the performance of the proposed method is evaluated with simulations. At first, we compare the proposed method with ED under different sampling numbers. Secondly, we evaluate the detection performance of the proposed method with the same total sampling numbers and different subband and sampling numbers. Finally, we compare with the methods in $[19,20,34]$.

In the following simulations, we consider a baseband signal with $B_{\text {tot }}$ bandwidth and decompose it into $K$ subbands, which means that the bandwidth of each subband is $B_{\text {sub }}=B_{\text {tot }} / K$. With an oversampling factor $N$, the sampling rate in each subband is then $2 B_{\text {sub }} N$. The parameters are summarized in Table 1 . Note that the simulation settings in Table 1 are only chosen to illustrate our purpose and some other values could be chosen.

\subsection{Performance comparison with ED}

In the first simulation, we examine the performance of the proposed method by comparison with the basic ED

Table 1 Simulation parameters

\begin{tabular}{ll}
\hline Parameter & Value \\
\hline Total bandwidth $B_{\text {tot }}$ & $100 \mathrm{MHz}$ \\
Bandwidth of each subband $B_{\text {sub }}$ & $5 \mathrm{MHz}$ \\
Number of subbands $K$ & 20 \\
Number of samples in each subband Q & $16 / 8$ \\
Oversampling factor $N$ & 100 \\
Sampling period $T_{S}=\frac{1}{2 B_{\text {sub }} N}$ & $0.001 \mu \mathrm{s}$ \\
\hline
\end{tabular}

method, which calculates the total energy in the full bandwidth as

$$
E=\sum_{k=1}^{K} \sum_{q=1}^{Q}\left|x_{q}^{(k)}\right|^{2}
$$

The noise power $\sigma^{2}$ is assumed to be known in ED method and in the proposed method. As performed in many practical schemes, the noise power can be estimated periodically when no signal is expected in the frequency band of interest. Note that in the comparison, the test statistic in ED method is approximated as Gaussian distributed thanks to the number of the total samples (e.g., $K Q>150$ ) and by using the central limit theorem.

Figure 4 presents the probability of detection $\left(P_{d}\right)$ of the proposed method with different sampling numbers $Q=16,8$, where the probability of false alarm $\left(P_{f a}\right)$ is set to 0.05 for different SNR. As can be seen, with the increase of SNR and for a given value of $Q$, the probability of detection of the proposed method rises up quickly which is better than the trend of the curves of ED method. For the proposed method, when $Q=8$, the probability of detection is 0.9796 at $-10 \mathrm{~dB}$. In order to clearly reveal the performance of the proposed method, the receiver operating characteristic (ROC) curves with different sampling numbers are shown in Fig. 5 when $\mathrm{SNR}=-15 \mathrm{~dB}$. It is obvious that the performance of the proposed method and ED is improved with the increase of the number of samples. When the probability of false alarm $P_{f a}$ is 0.1 with $Q=16,8$, the corresponding detection probabilities of the proposed method $P_{d}$ reach 0.9632 and 0.8155 , respectively.

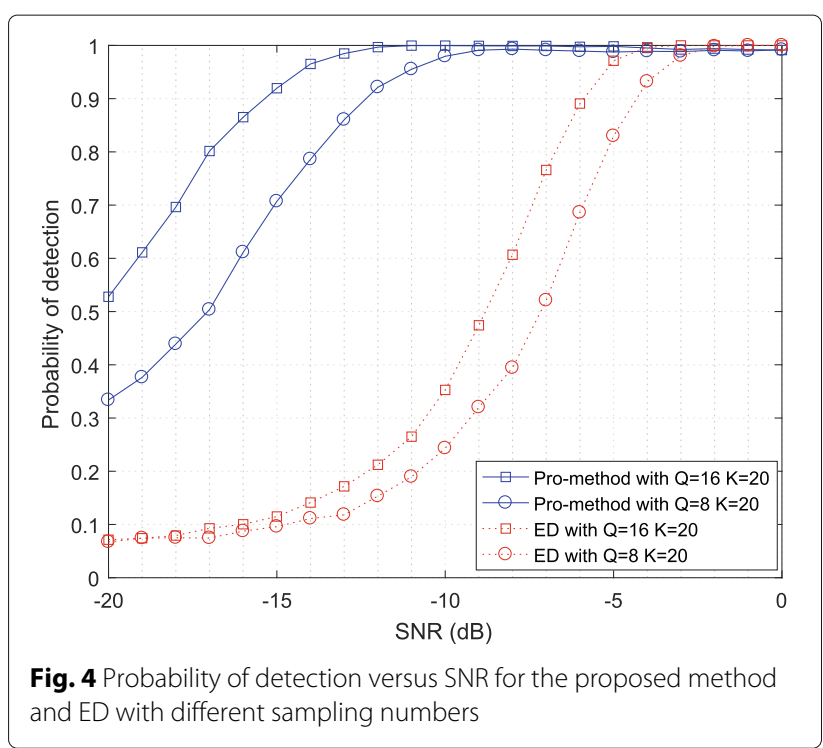




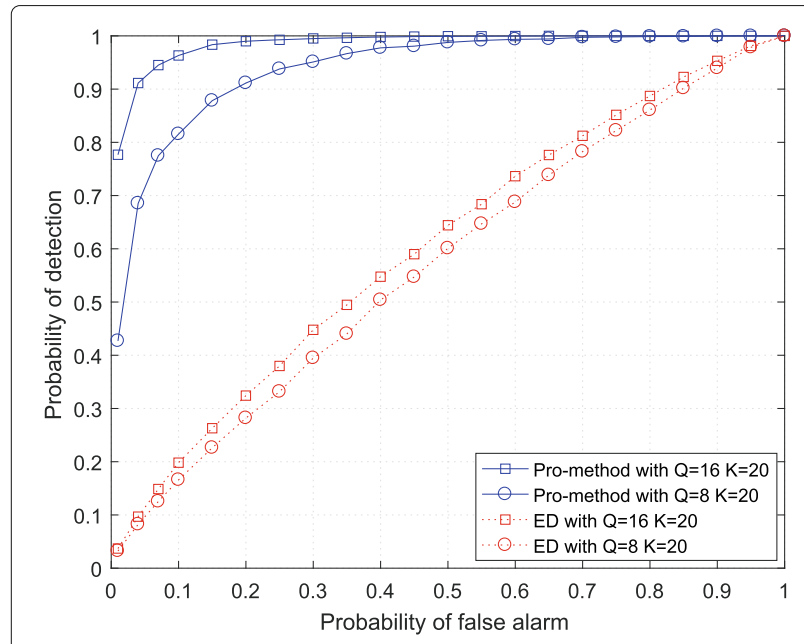

Fig. 5 ROC curves of the proposed method and ED with different sampling numbers at $\mathrm{SNR}=-15 \mathrm{~dB}$

\subsection{Performance analysis with different $Q$ and $K$}

In the second simulation, the detection performance of the proposed method with different $Q$ and $K$ is assessed. Figure 6 shows that $P_{d}$ of the proposed method goes up quickly with the increase of SNR, where $P_{f a}$ is 0.05 for different SNRs. As shown in Fig. 6, when the total number of samples is $K Q=600$, the detection performance of the proposed method is better than when $K Q=360$ and $K Q=120$. Moreover, for the same total number of samples, when the wideband is divided into more groups, a better performance can be obtained. As en example, for $\mathrm{SNR}=-15 \mathrm{~dB}$ and $K Q=360$, the magnified part of Fig. 6 shows that the detection probabilities are respectively $0.9501,0.9461$, and 0.9455 for $(Q=9, K=40)$, $(Q=12, K=30)$, and $(Q=18, K=20)$, respectively. This

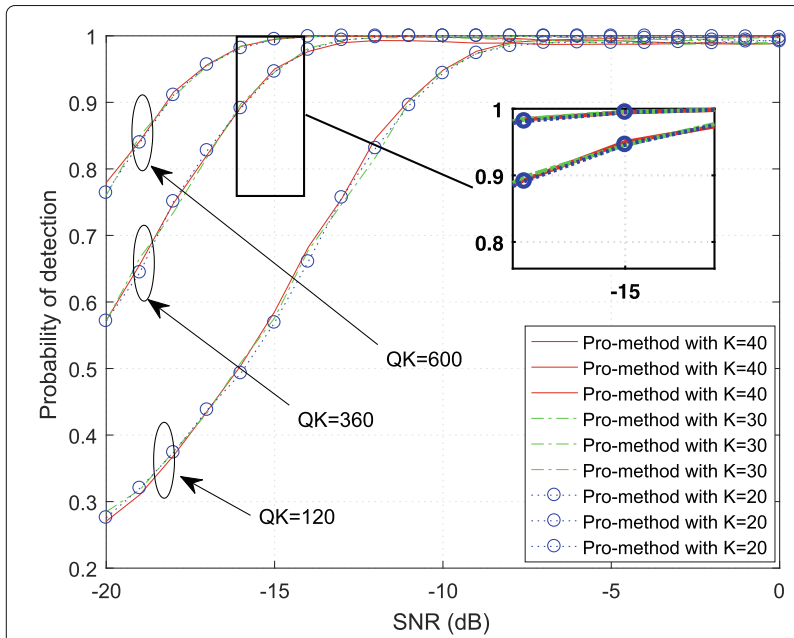

Fig. 6 Probability of detection versus SNR for the proposed method with $Q K=600,360,120$ and $K=40,30,20$ also verifies that a large $K$ results in a more reliable BPA estimation and finally gives a high detection probability. Moreover, the corresponding ROC curves are shown in Fig. 7 for SNR $=-15 \mathrm{~dB}$. It also verifies that a larger $K$ gives a better detection performance with the same total number of samples.

\subsection{Performance comparison with the methods in $[19,20$, 34] and ED method}

In the third simulation, we compare the performance of the proposed method with the GoF methods, such as the $\mathrm{AD}$ test-based method in [19], the $Z_{c}$ test-based method in [20], the decision fusion method in [34] and ED method with $Q=8, K=20$ for SNR $=-15 \mathrm{~dB}$. The $\mathrm{GoF}$ method is non-parametric which utilizes the distance between the empirical CDF of the received samples and the CDF of the noise distribution, to detect the presence of a signal. In [19], a blind spectrum sensing method based on the AD statistic and Student's $t$ distribution is proposed to outperform the energy detection. In [20], a non-parametric sensing scheme is proposed for the nonGaussian environment modelled by Middleton class A noise, where the PDF of the test statistics is approximated as log-normal and an expression of $P_{d}$ is derived. In [34], a semi-soft fusion scheme is proposed to achieve a trade-off between sensing performance and bandwidth cost. However, these methods are not designed for the small number of samples situation, nor wideband signal. As we can see in Fig. 8, the proposed method, the AD test-based method in [19], the $Z_{c}$ test-based method in [20], and the decision fusion method in [34] are better than ED when $Q=8$ and $K=20$ are used. When the probability of false alarm is equal to 0.1 , the detection probability of the proposed method is 0.8191 . This is about $21 \%$ better than the

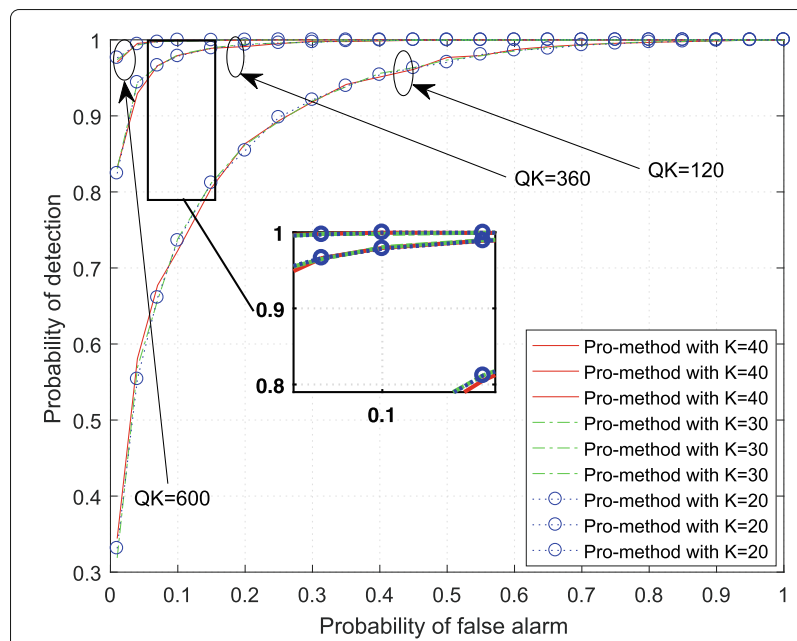

Fig. 7 ROC curves of the proposed method with $Q K=600,360,120$ and $K=40,30,20$ at $S N R=-15 \mathrm{~dB}$ 


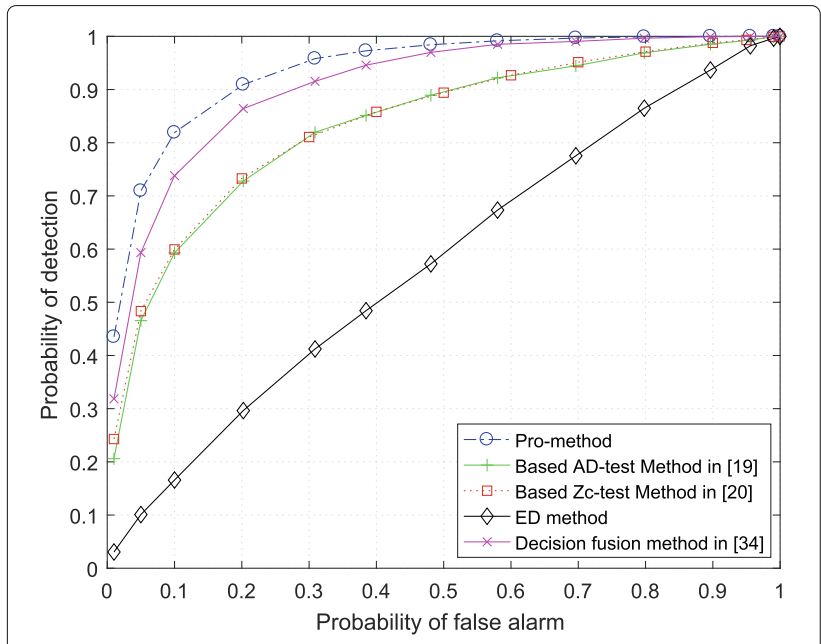

Fig. 8 ROC curves comparison among different spectrum sensing methods with $Q=8, K=20$ at $S N R=-15 \mathrm{~dB}$

detection probability of its counterparts (the GoF methods in $[19,20])$. Even comparing with the decision method proposed in [34], the detection probability of the proposed method has also about $8.1 \%$ increase.

In addition, in order to facilitate the comparisons with other methods, the number of samples required by the methods in $[19,20,34]$ and ED method to achieve a similar performance is determined. In Table 2, for the targeted performance $\left(P_{f a}, P_{d}\right)=(0.1,0.8191)$, it can be observed that the required number of samples for the methods in $[19,20,34]$ and ED method is 260, 260, 200, and 9900. However, the proposed method only requires 160 samples to achieve the same performance. In fact, reducing the number of samples without sacrificing the detection performance is a very attractive feature in practice, because it brings an economy in terms of computational burden, sensing time, and energy consumption.

\subsection{Discussion}

Considering that no prior knowledge about the channel is available, a simplified assumption of the constant amplitude in each subband is made in this paper. In order to support this assumption, some simulations are proposed to show that the method still works well even if the whole

Table 2 Required number of samples for different methods with same performance

\begin{tabular}{llll}
\hline Method & $P_{f a}$ & $P_{d}$ & Required number of samples \\
\hline ED method & 0.1 & 0.8191 & $9900(Q=495, K=20)$ \\
Method in [20] & 0.1 & 0.8191 & $260(Q=13, K=20)$ \\
Method in [19] & 0.1 & 0.8191 & $260(Q=13, K=20)$ \\
Method in [34] & 0.1 & 0.8191 & $200(Q=10, K=20)$ \\
Pro-method & 0.1 & 0.8191 & $160(Q=8, K=20)$ \\
\hline
\end{tabular}

bandwidth is not perfectly uniformly occupied. Let us consider amplitude $s^{(k)}$ in each subband as a Gaussian distribution with mean $\bar{s}=1$ and variance $\sigma_{s}^{2}$. Given that $\sigma_{s}^{2}=0$ corresponds to the constant amplitude assumption, we present the results with $\sigma_{s}^{2}=0.3$ and $\sigma_{s}^{2}=0.6$ in the following simulations, where $Q=16$ and $K=20$. As shown in Fig. 9, when $\sigma_{s}^{2}$ is increased from 0 to 0.3 and 0.6 , the proposed method still performs well compared to the classical ED technique. We can also see that the proposed method is not sensitive to the variation of power spectral density of the signal.

\section{Conclusion}

In this paper, we propose a wideband signal detection method for CR applications with limited resource. The advantage of the proposed technique compared to the traditional detection methods is that only small number of samples is required. In this work, the samples are fully exploited with Student's $t$ test which is more suitable to the small sample size case. New BPA functions based on the CDF of Student's $t$ distribution are constructed and used in the D-S fusion process, which improves the detection performance. Simulation results show that the proposed method can achieve a higher probability of detection than other compared methods with small sample size. The proposed method uses a small number of samples without sacrificing the detection performance, therefore brings an important economy in terms of computational burden, sensing time, and energy consumption. In the future work, multipath frequency selective channels between the PUs and the CR devices could be considered. As a result of such harsh environment (multipath with no line of sight and strong propagation loss), cooperative spectrum sensing techniques with small sample size will be developed. In

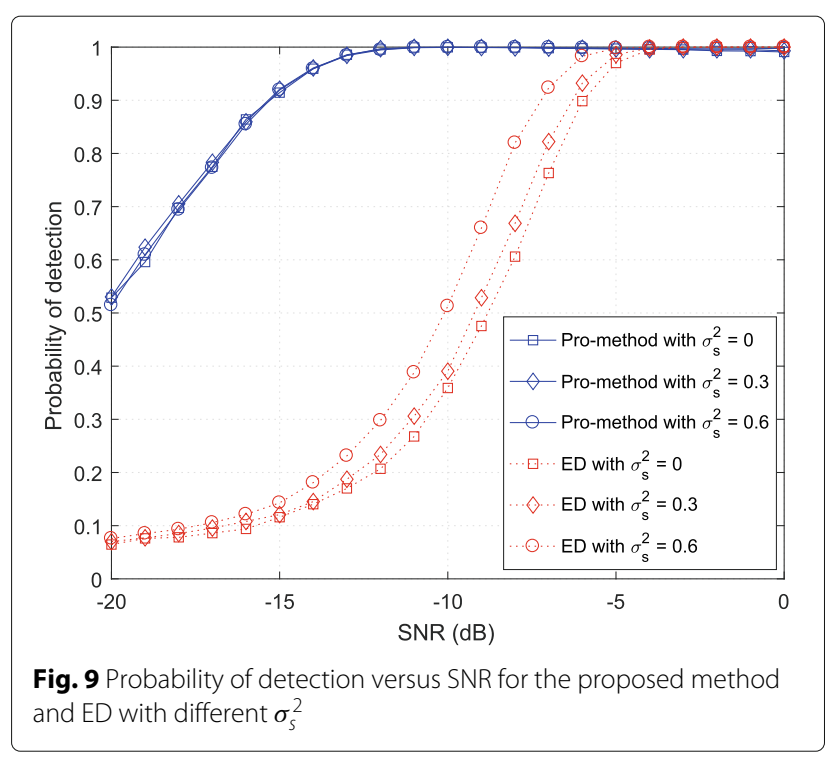


addition, due to an imperfect knowledge of the bandwidth especially at the edge of the spectrum or strong frequency attenuations through the channel, it may occur in practice that sometimes the band is not fully occupied when the signal is present. Although this situation is not considered in this study, a rejection of misleading subbands could be elaborated according to an evaluation of a similarity criteria among the subbands. This problem deserves to be taken into account in some future work.

\section{Abbreviations}

AWGN: Additive white Gaussian noise; BPA: Basic probability assignment; CDF: Cumulative distribution function; CR: Cognitive radio; D-S: Dempster-shafer; ED: Energy detection; GoF: Goodness of fit; PDF: Probability density function; SNR: Signal-to-noise ratio; SS: Spectrum sensing; WSN: Wireless sensor network;

\section{Funding}

This work is supported by the Medical Science and Technology Research Foundation of Guangdong under Grant No. B2018108, the Youth Creative Talent Project (Natural Science) of Guangdong under Grant No. 2017 KQNCX041 and the National Natural Science Foundation of China (NSFC) under Grant No. 61673260

\section{Availability of data and materials}

Please contact the author for data requests.

\section{Authors' contributions}

The work presented here was carried out in collaboration between all authors. The general idea was proposed by SM and PC. SM, YW, and JL designed and performed the simulations, and analyzed the results. SM wrote the paper. PC, $Y W$, and $J L$ revised the manuscript and provided many valuable suggestions. All authors read and approved the final manuscript.

\section{Ethics approval and consent to participate}

Not applicable.

\section{Consent for publication}

Not applicable.

\section{Competing interests}

The authors declare that they have no competing interests.

\section{Publisher's Note}

Springer Nature remains neutral with regard to jurisdictional claims in published maps and institutional affiliations.

\section{Author details \\ ${ }^{1}$ School of Medical Information Engineering, Guangzhou University of Chinese Medicine, No. 232, Waihuan East Road, Panyu District, 510006 Guangzhou, China. ${ }^{2}$ Institut d'Electronique et Télécommunications de Rennes (IETR), Université de Nantes, UMR CNRS 6164, Rue Christian Pauc BP 50609, 44306 Nantes, France. ${ }^{3}$ Shanghai Maritime University, No. 1550, Harbour Road, Pudong New Area, 201306 Shanghai, China. ${ }^{4}$ School of Automation, Guangdong University of Technology, No. 100, Waihuan West Road, Panyu District, 510006 Guangzhou, China.}

Received: 23 August 2018 Accepted: 28 December 2018 Published online: 16 January 2019

\section{References}

1. I. Kakalou, K. E. Psannis, P. Krawiec, R. Badea, Cognitive radio network and network service chaining toward $5 \mathrm{~g}$ : Challenges and requirements. IEEE Commun. Mag. 55(11), 145-151 (2017)

2. J. G. Prasad, N. S. Yeob, K. S. Won, Cognitive radio wireless sensor networks: Applications, challenges and research trends. Sensors. 13(9), 11196-11228 (2013)

3. A. A. Khan, M. H. Rehmani, M. Reisslein, Cognitive radio for smart grids: Survey of architectures, spectrum sensing mechanisms, and networking protocols. IEEE Commun. Surv. Tutorials. 18(1), 860-898 (2016)
4. G. Vijay, E. B. A. Bdira, M. Ibnkahla, Cognition in wireless sensor networks: A perspective. IEEE Sensors J. 11(3), 582-592 (2011)

5. H. Sun, A. Nallanathan, C. X. Wang, Y. Chen, Wideband spectrum sensing for cognitive radio networks: a survey. IEEE Wirel. Commun. 20(2), 74-81 (2013)

6. Y. Ma, Y. Gao, Y. C. Liang, S. Cui, Reliable and efficient sub-nyquist wideband spectrum sensing in cooperative cognitive radio networks. IEEE J. Sel. Areas Commun. 34(10), 2750-2762 (2016)

7. T. E. Bogale, L. Vandendorpe, L. B. Le, Wide-band sensing and optimization for cognitive radio networks with noise variance uncertainty. IEEE Trans. Commun. 63(4), 1091-1105 (2015)

8. E. Axell, G. Leus, E. G. Larsson, H. V. Poor, Spectrum sensing for cognitive radio : State-of-the-art and recent advances. Signal Proc. Mag. IEEE. 29(3), 101-116 (2012)

9. J. Font-Segura, G. Vazquez, J. Riba, Nonuniform sampling walls in wideband signal detection. IEEE Trans. Signal Process. 62(1), 44-55 (2013)

10. R. Gao, Z. Li, P. Qi, H. Li, A robust cooperative spectrum sensing method in Cognitive radio networks. IEEE Commun. Lett. 18(11), 1987-1990 (2014)

11. D. A. Guimaraes, G. P. Aquino, Resource-efficient fusion over fading and non-fading reporting channels for cooperative spectrum sensing. Sensors. 15(1), 1861 (2015)

12. Q. T. Vien, B. G. Stewart, H. Tianfield, H. X. Nguyen, Efficient cooperative spectrum sensing for three-hop cognitive wireless relay networks. IET Commun. 7(2), 119-127 (2013)

13. H. Urkowitz, Energy detection of unknown deterministic signals. Proc. IEEE. 55(4), 523-531 (2005)

14. S. K. Sharma, S. Chatzinotas, B. Ottersten, Eigenvalue-based sensing and snr estimation for cognitive radio in presence of noise correlation. IEEE Trans. Veh. Technol. 62(8), 3671-3684 (2013)

15. F. Lin, R. C. Qiu, Z. Hu, S. Hou, J. P. Browning, M. C. Wicks, Generalized fmd detection for spectrum sensing under low signal-to-noise ratio. IEEE Commun. Lett. 16(5), 604-607 (2012)

16. S. Wang, N. Rahnavard, in Information Sciences and Systems. Eigenvalue-based cooperative spectrum sensing with finite samples/sensors (IEEE, Princeton, 2012), pp. 1-5

17. W. Zhang, C.X. Wang, X. Tao, P. Patcharamaneepakorn, Exact distributions of finite random matrices and their applications to spectrum sensing. Sensors. 16(8), 1-22 (2016)

18. D. Denkovski, V. Atanasovski, L. Gavrilovska, Hos based goodness-of-fit testing signal detection. IEEE Commun. Lett. 16(3), 310-313 (2012)

19. L. Shen, H. Wang, W. Zhang, Z. Zhao, Blind spectrum sensing for cognitive radio channels with noise uncertainty. IEEE Trans. Wirel. Commun. 10(6), 1721-1724 (2011)

20. D. K. Patel, Y. N. Trivedi, Goodness-of-fit-based non-parametric spectrum sensing under middleton noise for cognitive radio. Electron. Lett. 51(5), 419-421 (2015)

21. L. Rugini, P. Banelli, G. Leus, Small sample size performance of the energy detector. IEEE Commun. Lett. 17(9), 1814-1817 (2013)

22. G. Shafer, A mathematical theory of evidence. (Princeton University Press, 1976)

23. J. Wang, S. Feng, Q. Wu, X. Zheng, Y. Xu, G. Ding, A robust cooperative spectrum sensing scheme based on Dempster-Shafer theory and trustworthiness degree calculation in cognitive radio networks. EURASIP J. Adv. Sig. Process. 2014(1), 1-12 (2014)

24. X. Xing, W. Wang, Z. Wang, K. Liu, in IEEE International Symposium on Microwave, Antenna, Propagation and Emc Technologies for Wireless Communications. Weighted cooperative spectrum sensing based on $\mathrm{d}$-s evidence theory and double-threshold detection (IEEE, Chengdu, 2013), pp. 145-149

25. N. Nguyen-Thanh, I. Koo, Evidence-theory-based cooperative spectrum sensing with efficient quantization method in cognitive radio. IEEE Trans. Veh. Technol. 60(1), 185-195 (2011)

26. Y. Han, Q. Chen, J. X. Wang, in Vehicular Technology Conference. An enhanced $d$-s theory cooperative spectrum sensing algorithm against ssdf attack (IEEE, Yokohama, 2012), pp. 1-5

27. S. Men, P. Charge, S. Pillement, in IEEE International Conference on Communication Workshop. A robust cooperative spectrum sensing method against faulty nodes in cwsns (IEEE, London, 2015), pp. 334-339

28. Q. Chen, A. Whitbrook, U. Aickelin, C. Roadknight, Data classification using the dempster-shafer method. J. Exp. Theor. Artif. Intell. 26(4), 493-517 (2014) 
29. S. Men, P. Charge, S. Pillement, Cooperative spectrum sensing with small sample size in cognitive wireless sensor networks. Wirel. Pers. Commun. 96(2), 1871-1885 (2017)

30. X. Liu, M. Jia, Z. Na, W. Lu, F. Li, Multi-modal cooperative spectrum sensing based on dempster-shafer fusion in $5 \mathrm{~g}$-based cognitive radio. IEEE Access. 6(99), 199-208 (2018)

31. K. Arshad, K. Moessner, Robust spectrum sensing based on statistical tests. IET Commun. 7(9), 808-817 (2013)

32. E. L. Mokole, T. K. Sarkar, in International Conference on Electromagnetics in Advanced Applications. Introduction to ultrawideband theory/technology/systems (IEEE, Cairns, 2016), pp. 768-771

33. F. Catherine, E. Merran, H. Nicholas, P. Brian, Statistical distributions (2011)

34. P. Verma, B. Singh, On the decision fusion for cooperative spectrum sensing in cognitive radio networks. Wirel. Netw. 23(7), 1-10 (2016)

\section{Submit your manuscript to a SpringerOpen ${ }^{\circ}$ journal and benefit from:}

- Convenient online submission

Rigorous peer review

- Open access: articles freely available online

- High visibility within the field

- Retaining the copyright to your article

Submit your next manuscript at $>$ springeropen.com 